\title{
La normativa comunitaria actualmente vigente en el llamado Derecho europeo de la Seguridad Social
}

\author{
Víctor Fernando Manrique López \\ Catedrático de Derecho del Trabajo \\ Universidad de Deusto
}

Resumen: La realidad comunitaria en materia de protección social viene marcada por la necesidad de hacer frente a las dificultades que pueda suscitar el desplazamiento de un país a otro dentro del mercado de trabajo comunitario, que se resumen en la implicación, respecto de un mismo trabajador, de más de un sistema de Seguridad Social (los cuales aunque pueden llegar a tener estructuras semejantes, son fruto de la evolución económica, social y política seguida en cada país y en cada sociedad), y en la descoordinación de los sistemas afectados de Seguridad Social, todo lo cual ha obligado a la Comunidad Europea a intentar su solución o, mejor dicho, a la minoración de las consecuencias de la libre circulación de los trabajadores.

Si bien es cierto que el artículo 51 del Tratado Constitutivo de la CEE, establecía que el Consejo, a propuesta de la Comisión, adoptaría en materia de Seguridad Social, las medidas necesarias para el establecimiento de la libre circulación de los trabajadores, no lo es menos, que a fecha de hoy, ni los Tratados Fundacionales, ni el Reglamento 1408/71 y sus ampliaciones, han creado un sistema europeo de seguridad social, uniforme y de directa aplicación para todos los Estados miembros, sino que se han limitado a remover las trabas a la libre circulación de los trabajadores y para ello a coordinar las legislaciones internas mediante la aplicación de unos principios específicos (igualdad de trato, no discriminación respecto de los trabajadores nacionales, y libre circulación).

En el futuro, dado el grado de interdependencia existente entre las economías de los Estados miembros que se deriva de la U.E., todo parece indicar que la protección social evolucionará, pasando a ser cada vez más un tema de interés común para los Estados miembros a los que interesa hallar cuál debe ser el planteamiento común, al encontrarse todos los Estados con problemas semejantes como son el envejecimiento demográfico y exigencia común de niveles de cobertura más adecuados. social.

Palabras clave: Derecho comunitario, reglamentos comunitarios, seguridad

Abstract: Social Protection as regards European Community's affairs, has been traditionally characterised by the need to overcome the difficulties that may arise owing to workers' mobility within the European common market, it falling short of individuals being subject to more than one Social Security System (which, notwithstanding the possibility of having similar structures, are the 
natural consequence of existing economic, political and social backgrounds, in each country and society), and a lack of coordination existing, amongst the Social Security Systems in dispute. All of which, has made the European Community need to try and find a solution to this problem, or, more appropriately, to lessen its consequences.

Whereas article 51 of the founding treaty of the EEC, established that the Council, backed by a proposal from the Commission, was to adopt all necessary measures needed, as regards Social Security, to enable the envisaged free movement of workers, as of today, neither the Founding Treaties, or Regulations 1408/71 and its extensions, establish a homogeneous Social Security System, directly applicable to its Member States. Instead, a policy aimed, and limited to, the removal of the barriers affecting the free movement of workers, has been sought through the coordination of internal legislation, and the application of specific principles (equal treatment, non discrimination from other EU Member State nationals', and free movement).

In the future, given the degree of interdependence reached amongst the economies of the European Member States, everything seems to point in the direction of social protection evolving towards an ever growing area of common interest, where these Member States must seek the manner of reaching and agreeing on a common position, over what was until now, a de facto relegated issue, since all of them will be facing similar problems such as aging of its population, and the common need for more adequate levels of protection.

Kew words: European law, regulations, social security.

Sumario: 1. Introducción. - 2. Origen y modificaciones de los Reglamentos Comunitarios en materia de Seguridad Social.-3. Vigencia de los Convenios Bilaterales. - 4. Síntesis de los Reglamentos Comunitarios: 4.1. Ámbito de aplicación subjetivo. 4.2. Ámbito material. 4.3. Contenido.-5. El Reglamento 883/2004: 5.1. Régimen Vigente.-6. Perspectivas de futuro.

\section{Introducción}

Debo empezar esta ponencia afirmando algo que es obvio, es un reto trasladar a estos folios lo que el enunciado hace presumir. Tal y como reza el título se supone que esta ponencia se divide en dos etapas. Una, la de situar el contexto actual de los reglamentos comunitarios y otra, cargada más de deseos, la de aventurar el posicionamiento de Europa sobre los ordenamientos de la Seguridad Social y qué modificaciones legales serían deseables.

¿Cuál es el presente de los Reglamentos Comunitarios? Analizarlo exige tener en cuenta su origen y modificaciones. 


\section{Origen y modificaciones de los Reglamentos Comunitarios en materia de Seguridad Social}

El artículo 51 del Tratado Constitutivo de la entonces CEE dispone que el Consejo, a propuesta de la Comisión, adoptará en materia de Seguridad Social, las medidas necesarias para el establecimiento de la libre circulación de los trabajadores, creando en especial, un sistema que permita garantizar a los trabajadores migrantes y sus derechohabientes: a) la acumulación de todos los periodos tomados en consideración por las distintas legislaciones nacionales para adquirir y conservar el derecho a las prestaciones sociales, así como para el cálculo de éstas; b) el pago de las prestaciones a las personas que residan en los territorios de los Estados miembros.

En aplicación del antiguo artículo 51 se dictaron los Reglamentos 3/58, de 25 de septiembre; 4/58, de 3 de diciembre, Reglamentos de Coordinación que fueron sustituidos por los reglamentos 474/72 y 1408/71 y que han sufrido sucesivas modificaciones (Reglamento 2001/83, de 2 de junio, 1248/92, de 30 de abril, 118/97, de 2 de diciembre, 1290/97, 1223/98, 859/03, etc.) y que serán sustituidos por el Reglamento 883/04, de 29 de abril de 2004, cuya vigencia está condicionada a la entrada en vigor el Reglamento de aplicación.

Llegados a este punto, a modo de breve introducción, y a los efectos de lograr abordar con éxito el objeto de la presente ponencia, resulta absolutamente necesario realizar una aproximación al reglamento 1408/71, y a la historia reciente del mismo.

Como punto de partida de esta historia reciente del Reglamento 1408/71, resulta de obligada referencia la propuesta específica de la Comisión, realizada en 1997, para la extensión de dicho Reglamento 1408/71 a los nacionales de Terceros Estados. Dicha propuesta, fue planteada por los servicios jurídicos de la propia Comisión, con base jurídica en los arts. 18, 42 y 308 del Tratado de la Comunidad Europea, que sin embargo encontró en el Consejo serios problemas, en tanto que los servicios jurídicos del mismo estimaron que el planteamiento de la propuesta de modificación debía encontrar su fundamentación en el art. 63.4 del Tratado, y no en los que había manifestado la Comisión en su propuesta.

Como consecuencia de esto, y ante los problemas que se habían suscitado con su propuesta original de 1997, la Comisión planteó en 1998 una nueva propuesta, esta vez global, referida al Reglamento 1408/71, por la que se buscaba por una parte impulsar la simplificación del mismo, dada las nuevas necesidades de aplicación, y por otro lado, dar salida a la fallida tentativa anterior de extensión del mismo a los nacionales de terceros Estados. 
Dicha propuesta, ante la ya evidentes y cada vez más acuciantes necesidades de la UE, contó esta vez con un Mandato del Consejo Europeo de Estocolmo para elaborar los parámetros de Modernización del Reglamento 1408/71.

El punto de inflexión, no obstante, se produce el 8 de octubre de 2001, cuando el Consejo de Empleo y Política Social da el mandato al Comité de Representantes Permanentes (COREPER) para encontrar la base jurídica adecuada.

Será por tanto durante la Presidencia española del Consejo de la U.E. (primer semestre del 2002), donde habiéndose optado por una tramitación en paralelo del Reglamento 1408/71, se llevará a cabo la negociación en el Consejo de la propuesta General, esto es sin nacionales de terceros Estados, sobre la base jurídica tradicional T.C.E. arts. 18, 42 y 308, y por otro lado, se buscará en exclusiva, el acuerdo en relación con la extensión del Reglamento 1408/71 a nacionales de terceros Estados, sobre la base jurídica del art. 63.4 del T.C.E.

Todo lo cual desembocará en un acuerdo político respecto de ambas negociaciones, en relación con los Títulos I y II del Reglamento 1408/71, alcanzado bajo la propia Presidencia española, que será la base para lo que después habrían de ser los Reglamentos 883/04 y 859/03, sobre simplificación, y extensión de aplicación a nacionales de terceros Estados, respectivamente.

No obstante lo anterior, en la materia que tratamos, ni los Tratados Fundacionales ni el Reglamento 1408/71 han creado un sistema europeo de Seguridad Social, uniforme para todos los Estados miembros, que fuera de directa aplicación a todos ellos, sino que se han limitado a remover las trabas a la libre circulación de los trabajadores y para ello a coordinar las legislaciones internas mediante la aplicación de unos principios (igualdad de trato, no discriminación respecto de los trabajadores nacionales y libre circulación) que, además, ni siquiera están todos recogidos en el derecho comunitario originario (tan sólo en la Carta de Derechos Fundamentales de la Unión Europea de 2000), sino que se introducen en los Reglamentos y en la jurisprudencia comunitaria.

Las dificultades de armonización y coordinación entre los diferentes sistemas de Seguridad Social existentes en los países miembros de la Comunidad Europea vienen dadas por un lado, porque los sistemas de protección social, aunque pueden llegar a tener estructuras semejantes, son fruto de la evolución cultural, social y política seguida en cada país y en cada sociedad, que han tenido circunstancias y experiencias diferentes.

Por otro lado la dificultad va más allá al afectar las diferencias a los propios modelos existentes, contributivos y universalistas, aunque lo único cierto es que ningún sistema es puramente contributivo ni puramente universal. Incluso los sistemas se han ido transformando y evolucionando (es 
ejemplo la protección por asistencia sanitaria que marca la evolución hacia fórmulas propias de los sistemas universalistas).

Pese a ello, la necesidad de hacer frente a las dificultades que pueda suscitar el desplazamiento de un país a otro dentro del mercado de trabajo comunitario, que se resumen en la implicación de más de un sistema de Seguridad Social respecto de un mismo trabajador y en la descoordinación de los sistemas implicados de Seguridad Social han obligado a la Comunidad Europea a intentar su solución o, mejor dicho la minoración de las consecuencias de la libre circulación de los trabajadores.

La incorporación de España a las Comunidades Europeas, autorizada por Ley orgánica 10/85, de 2 de agosto y, efectiva desde el 1 de enero de 1986, determinó la atribución a éstas del ejercicio de las competencias derivadas de la propia C.E. y, por tanto, el reconocimiento a las fuentes comunitarias de una posición de privilegio dentro del ordenamiento jurídico español.

La aplicación de lo antes expuesto determina que, por un lado, los órganos administrativos y, los jueces españoles, en la revisión de los actos de aquéllos, sean competentes para aplicar e interpretar el derecho comunitario vinculante, esto es el Derecho comunitario originario, los Reglamentos y, con las debidas matizaciones, las Directivas y las Decisiones. Por otro lado, y, para la teórica garantía de coordinación, el Tribunal de Justicia de las Comunidades Europeas se alza en jurisdicción única, originaria, propia y exclusiva encargada de interpretar y aplicar el derecho comunitario, ya sea mediante la solución de los recursos que son promovidos (por los Estados, las Instituciones y los particulares), ya sea en la solución de las cuestiones prejudiciales que cualquier órgano jurisdiccional de un Estado miembro lo estime necesario.

El análisis de la situación presente nos dice que la aplicación de los Reglamentos Comunitarios en procedimientos de Seguridad Social española se ha visto incrementada en los últimos años, debido a la masiva emigración de trabajadores españoles hacia países hoy integrados con España en la UE $\mathrm{y}$, se ha traducido en el correlativo incremento de la litigiosidad en esta materia, dada la intrínseca dificultad interpretativa de los Reglamentos Comunitarios en materia de Seguridad Social. Este incremento, sin embargo, no ha tenido igual proporción respecto del planteamiento de las llamadas cuestiones prejudiciales. Apenas una docena en la última década, relativas a la materia que nos ocupa, que se plantearon en relación con la interpretación del Reglamento 1408/71 del Consejo, relativo a la aplicación de los regímenes de Seguridad Social a los trabajadores por cuenta ajena y a sus familias que se desplazan dentro del ámbito comunitario.

En este sentido se han planteado cuestiones prejudiciales tan sólo sobre la incompatibilidad entre pensión de jubilación y trabajo en la función 
pública resultante de la aplicación de la LGPE para 1984; sobre la aplicación de la normativa española sobre subsidio de desempleo en el caso de emigrantes retornados mayores de cincuenta y dos años y, la interpretación de las reglas sobre el cálculo de la base reguladora y cuantía de las pensiones de invalidez y jubilación reconocidas, igualmente a emigrantes retornados.

Desde que se promulgó la normativa comunitaria en materia de Seguridad Social, el Tribunal de Justicia de las Comunidades Europeas ha emitido más de 500 sentencias en torno a su interpretación, la mayoría de ellas a favor de los trabajadores migrantes y sus familiares. Esta cifra demuestra la función esencial del tribunal en la interpretación de las dudas sobre el alcance y su extensión de la normativa comunitaria, su aplicación a los casos concretos y su interpretación a la luz de la ley nacional.

\section{Vigencia de los Convenios Bilaterales}

Pues bien, las consecuencias prácticas de la integración española nos plantea, como primera cuestión y como aplicadores de las normas, la derogación o no de los Convenios Bilaterales que estaban en vigor al ingresar España en la CEE.

Del tenor literal de la norma se desprende que la suscripción de los Reglamentos Comunitarios conlleva la inaplicación de los Convenios Bilaterales que tenía suscrito el Estado español. Así, el art. 6-a del Reglamento 1408/71 dispone que los preceptos que en él se recogen sustituyen a cualquier Convenio de la Seguridad Social que vincule a dos o más Estados miembros. Es decir, establece la sustitución de todos los convenios de los Estados miembros.

El citado precepto fue interpretado por el TJCE en la sentencia de 7 de junio de 1973 (asunto Walder) en el sentido de que el principio de sustitución de las disposiciones de los Convenios de Seguridad Social concertados entre Estados miembros tiene un alcance imperativo que no admite excepciones fuera de los casos expresamente previstos.

Esta que fue la tesis que, por la literalidad de la norma vino aplicando de forma uniforme el Tribunal Supremo, varía sustancialmente cuando se comprueba que los derechos de los trabajadores se ven en alguna materia minorados de aplicar los Reglamentos Comunitarios. Nos referimos por ejemplo al cálculo de la base reguladora (máximas, medias o mínimas) o, a la aplicación de la bonificación de edad a trabajador que sirve en embarcaciones pertenecientes a la UE (según prevé el art. 2 de la Orden de 17 de noviembre de 1983 condicionado a la existencia de convenio con aquel país). 
El TSJ, sin embargo, apartándose de la doctrina sentada en el caso Walder (que atribuía imperatividad absoluta al Reglamento) dicta la sentencia de 7-2-91 (asunto Ronfeld) afirmando la no sustitución de los Convenios o tratados precedentes a la integración, cuando éstos son más favorables para los trabajadores que la normativa comunitaria. Posteriormente, la sentencia de 9 de noviembre de 2000 (TJCE 2000, 268), asunto Thelen, ratifica la doctrina establecida en las anteriores sentencias, Naranjo Arjona (TJCE 1997, 203), Thevenon (TJCE 1995, 198) y Grajera (TJCE 1998/319), en el sentido de que el art. 6 del Reglamento 1408/71 no se opone a la aplicación de las disposiciones de un Convenio entre Estados, más favorable.

La aplicación en España de la sucesión de las doctrinas antes plasmada supuso, en la materia de aplicación de bonificaciones a los trabajadores embarcados en buques de bandera comunitaria, que la Entidad Gestora no aplicara la bonificación prevista en la Orden de 17-11-83 que exige la existencia de Convenio suscrito con aquel país, fundamentándolo en la sustitución de los Convenios por los Reglamentos (Circular 12/90, de 28 de noviembre). Por el contrario, la Entidad Gestora, en la materia correspondiente a la forma de cálculo de la base reguladora, no aplicó los Reglamentos Comunitarios, el 1408/71 en su redacción previa a la reforma operada por Reglamento 1248/92, que en su artículo 47 hace referencia a un régimen de cálculo de las prestaciones de invalidez fundado en una base media de cotización, por el contrario aplicó la Entidad bases mínimas. En ambos casos el Tribunal Supremo modificó la interpretación realizada, si bien respecto de la forma de cálculo de la base reguladora, la modificación del Reglamento 1408/71 por el 1248/92 todavía dio lugar a un nuevo cambio de doctrina (bases remotas correspondientes a los años inmediatamente anteriores al pago de la última cotización a la Seguridad Social española, STJCE 17-12-98), eso sí manteniendo la posibilidad de acudir al Convenio Bilateral (bases medias según jurisprudencia del Tribunal Supremo, pese a que el art. 25 se remite a las bases de cotización vigentes en España...para los trabajadores de la misma categoría profesional que la persona interesada) de resultar más beneficiosa y, siempre condicionado que los trabajadores hubieran ejercido su derecho a la libre circulación después de la entrada en vigor del mencionado Reglamento (1-1-86 para España) como estableció la sentencia Thévenon de fecha 9-1-95.

En definitiva y aun cuando parece definitivamente zanjada la cuestión, no nos cabe duda que no cesarán los procedimientos instados por quienes trabajaron en países comunitarios en fecha posterior a enero de 1986 opondrán la vulneración de su derecho a la libre circulación. No en vano en la comparación entre la fórmula de cálculo del Reglamento (1243/92) y el Convenio bilateral, finalmente el Tribunal Supremo reconoció en la sentencia de 10 de marzo de 1999 que el reglamento es menos favorable que el 
Convenio aunque, eso sí, mantiene pese al tenor del Convenio, la doctrina de cálculo según las bases medias de cotización vigentes en España para los trabajadores de la misma categoría profesional que la persona interesada.

\section{Síntesis de los Reglamentos Comunitarios}

\section{1. Ámbito de aplicación subjetivo}

Originariamente el Reglamento 1408/71 limitaba su campo de aplicación a los nacionales de un Estado miembro de la Unión Europea, con la única excepción de los apátridas y refugiados que residan en un Estado miembro.

Sin embargo en el ámbito de aplicación de los reglamentos se incluyen también Islandia, Noruega y Liechtenstein, cuyos nacionales gozan del derecho a la libre circulación en virtud del Acuerdo sobre el Espacio económico Europeo de 1992. Se extiende a Suiza por el Acuerdo sobre libre circulación de personas entre la Comunidad Europea y sus Estados miembros y la Confederación Suiza de 21 de junio de 1999.

Se aplica también a los territorios asociados a la Comunidad por su vinculación con algún Estado comunitario (Groenlandia). Y a los Estados que tienen un Acuerdo de Cooperación con la UE (Argelia, Turquía) para los que la sentencia del TJCE de 31 de enero de 1991 (asunto Kziber 18/90), entendió que son de aplicabilidad directa.

El ámbito de aplicación se modifica con el Reglamento 859/2003, de 14 de mayo extendiendo los derechos concedidos por las normas comunitarias a «los nacionales de terceros países que, debido únicamente a su nacionalidad, no estén cubiertos por la mismas, así como a los miembros de sus familias y a sus supervivientes, siempre que se encuentren en situación de residencia legal en el territorio de un Estado miembro y siempre que se encuentren en una situación en la que todos los elementos no estén situados en el interior únicamente de un solo Estado miembro». En consecuencia, los extranjeros tienen derecho a la totalización de los periodos de cotización y/o residencia acreditados en todos los Estados a los que se aplica el reglamento Comunitario, excepto Dinamarca.

Esto se debe a que la propuesta inicial de la Comisión (1997) relativa a la ampliación a nacionales de terceros Estados del campo de aplicación del Reglamento 1408/71, tomó como base jurídica de su propuesta los arts. 18, 42 y 308 del T.C.E., y, sin embargo, el Servicio Jurídico del Consejo consideró más adecuado como base jurídica de dicha propuesta específica, el art. 63.4 del T.C.E (Título IV: visados, asilo, inmigración y otras políticas sobre libre circulación de personas), con las correspondientes consecuencias jurídicas, 
cuales son la afectación del Protocolo 4 de los Tratados (necesidad de Opting-in del Reino Unido e Irlanda: siendo el Opting-in el procedimiento de declaración de voluntad en virtud del cual se manifiesta el deseo de un Estado que cuenta con potestad para ello - Reino Unido e Irlanda, en este caso- de quedar obligado por el contenido de las negociaciones de la normativa comunitaria y el resultado final de la misma - Reglamento, Directiva, ...- que de otra forma no le afectaría) y del Protocolo 5 (posibilidad de que Dinamarca no participe en virtud del Opting-out: procedimiento de declaración de voluntad, en virtud del cual un Estado - Dinamarca, en este caso- expresa su deseo de quedar al margen de la normativa comunitaria, en aquellas materias en las que cuenta con potestad para ello, normativa que de otra forma le afectaría plenamente).

Surge así el dilema en cuanto a la coordinación de las negociaciones a 12,13 o 14 Estados miembros (a partir de finales de enero comenzó formalmente el periodo de 3 meses de Opting-in, y consulta al Parlamento Europeo, que Reino Unido e Irlanda finalmente ejercitaron), y que dieron lugar al acuerdo político en cuanto al campo de aplicación Personal y Material (Título I y II de la propuesta de la Comisión), alcanzado en la Presidencia española.

El Reglamento 883/04 mantiene la extensión antes citada al mantener en vigor los efectos jurídico del Reglamento 859/03.

\section{2. Ámbito material}

Desde el punto de vista material los reglamentos se aplican tanto a las prestaciones contributivas como a las no contributivas, en lo relativo a las siguientes prestaciones: enfermedad, maternidad, invalidez, vejez, supervivencia, accidente de trabajo, enfermedad profesional, subsidios de defunción, desempleo, prejubilación y prestaciones familiares. No se aplica ni a la asistencia social y médica ni a la previsión social complementaria.

Los regímenes complementarios o suplementarios se extienden por todos los países de la Unión Europea ya tengan sistemas contributivos, ya asistenciales. En los Reglamentos 1408/71 y 574/72 hay un deficitario tratamiento de los sistemas de previsión complementaria o voluntaria, lo que tiene un especial interés de cara a garantizar el derecho a la libre circulación de trabajadores migrantes. Sin embargo, los intentos de regulación tendentes a facilitar el principio de igualdad de trato y la transferencia de los derechos causados han sido varias y entre ellas:

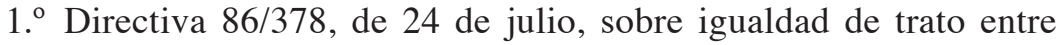
hombres y mujeres en los regímenes profesionales de Seguridad Social. 
2. ${ }^{\circ}$ Directiva 77/187, de 14 de febrero, sobre la aproximación de las legislaciones de los Estados miembros relativas al mantenimiento de los derechos de los trabajadores en caso de traspasos de empresas, de centros de actividades o de partes de centros de actividad, que excluye de su ámbito de aplicación la conservación de los derechos de los trabajadores derivados de los regímenes complementarios profesionales o interprofesionales que existan con independencia de los regímenes de Seguridad Social de los Estados miembros.

3..$^{\circ}$ Directiva 80/987, de 20 de octubre, sobre la aproximación de las legislaciones de los Estados miembros relativa a la protección a los trabajadores asalariados en caso de insolvencia del empresario, en lo que se refiere a garantías y protección de sus derechos adquiridos o en curso de adquisición, respecto de las prestaciones de vejez y supervivencia.

Por tanto y como anticipación de las perspectivas de futuro, nos encontramos ante una materia que debe ser regulada a fin de proteger a los trabajadores migrantes contra la pérdida de derechos de Seguridad Social complementaria. Se hace preciso que en los Reglamentos Comunitarios se fijen unas exigencias mínimas para llegar a unas disposiciones, también mínimas, pero comunes a los Estados miembros, que tengan como finalidad la de proteger y garantizar los derechos adquiridos y se garantice el principio de igualdad de trato.

\subsection{Contenido}

La coordinación, finalidad de los Reglamentos Comunitarios, se plasma en diversas técnicas o principios destinados a lograr su efectividad, principios y técnicas comunes a los Reglamentos 1408/71 y 574/72 e, igualmente en el reglamento 883/2004 y éstos son:

El principio de igualdad de trato y de reciprocidad en el tratamiento de los nacionales de otros Estados miembros, respecto de los propios nacionales de acogida.

El principio de unicidad de la legislación aplicable. Será sólo una la legislación competente para determinar los derechos de Seguridad Social del migrante. La regla general es la aplicación de la Ley del lugar de prestaciones de servicios en el momento del hecho causante, salvo excepciones tales como: será el lugar de residencia en el caso de desplazamiento temporal. Será el de la sede de la empresa en el caso de que los trabajadores desempeñen su actividad en más de un Estado.

La exportación de prestaciones, con la finalidad de mantener derechos adquiridos generados en un país cuando se traslade a otro. Principio inaplicable a la prestaciones no contributivas. 
Conservación de derechos en curso de adquisición, principio que se manifiesta en la totalización de los periodos de cotización o residencia acreditados en cualquiera de los Estados a los que se aplica el reglamento.

Por último el pago a prorrata, como reparto en el coste de la prestación a la vista de los periodos de cotización o, en su caso, residencia, en otro Estado. Aunque el reconocimiento de la prestación corresponde a un solo Estado, los Estados que han sido tenidos en cuenta para el cálculo de la citada prestación deberán contribuir al pago de la pensión en la parte proporcional a los periodos. Principio y regla inaplicable a las prestaciones no contributivas.

La coordinación comunitaria se resuelve de diferentes maneras porque para algunas prestaciones se resuelve en una totalización sin prorrateo; para otras en prorrateo sin totalización y, para otras, se resuelve con totalización con prorrateo. El último caso (totalización con prorrateo) no es habitual aun cuando es el que rige en el caso de las pensiones de jubilación y en parte de las de invalidez.

La totalización sin prorrateo existe para la invalidez entre países de legislación «A» (España, Francia, Bélgica, Holanda, Reino Unido) con las perversas consecuencias de que, según el país donde quede inválido el trabajador, percibirá la pensión en cuantías abismalmente distintas respecto de las que hubiera percibido si el accidente hubiera ocurrido en otro país. Paga un solo país pero debe sumar y totalizar los periodos de seguro cumplidos en otros países miembros. Se trata de una fórmula desequilibrada, que deberá ser revisada a fin de conciliar los principios comunitarios de igualdad, y de equilibrio financiero, al asumir la totalidad de pago el Estado miembro.

\section{El Reglamento 883/2004}

\subsection{Régimen Vigente}

Publicado el Reglamento 883/04, el pasado 30 de abril, denominado sobre la coordinación de los sistemas de Seguridad Social y, aunque se indicaba entraba en vigor a los 20 días de su publicación, sin embargo el art. 91 condicionaba su entrada en vigor a la fecha de entrada en vigor del Reglamento de aplicación.

El reglamento citado viene inspirado por dos grandes fines: a) perfeccionar los principios y técnicas a fin de mejorar la coordinación entre los distintos sistemas nacionales de Seguridad Social. Y b) abrir nuevas vías de homogeneización de los sistemas de Seguridad Social.

Las razones que han justificado la reforma de 2004 vienen dadas no sólo por el cambio de la realidad social desde la década de los 70 , fecha de los reglamentos en vigor y, la existencia de jurisprudencia y doctrina que 
provoca la necesidad de ajustar la norma. En concreto en el preámbulo del Reglamento considera que: «el Reglamento ha sido modificado en numerosas ocasiones para reflejar no sólo la evolución experimentada a nivel comunitario sino además por el contenido de las sentencias dictadas por el Tribunal Superior de Justicia de la Comunidad Europea y además por los cambios que se han ido produciendo en las legislaciones nacionales».

El reglamento, elaborado en aras de la transparencia y claridad, tiene como gran objetivo la simplificación de las reglas de coordinación.

Las modificaciones más relevantes introducidas son las siguientes y a tal efecto:

1. Respecto de la determinación de la legislación aplicable, el art. 11.1 del reglamento de 2004 establece que «sólo estarán sometidos a la legislación de un único Estado miembro». La fijación de cual viene establecida en el art. 13 remitiéndose a la legislación del Estado en el que se realiza la actividad productiva. Esta regla no es aplicable en tres supuestos que son los referidos a:

a. Trabajadores desplazados temporalmente al extranjero, supuesto ya contemplado en el Reglamento 1408/71 si bien se amplía el plazo a 24 meses. Empleado de transporte en los que por la propia dinámica de la prestación de servicios resulta inaplicable la regla de aplicación de la legislación que corresponde al lugar de prestación de la actividad, para tenerla que fijar una completa. Por último, los sujetos que desarrollan su actividad en varios Estados, en cuyo caso se estará a la legislación del Estado residencia del trabajador.

b. Universalización del ámbito de aplicación subjetivo. El artículo 1 establece que el presente reglamento se aplicará a las personas que estén o hayan estado sometidas a la legislación de Seguridad Social en alta mar.

2. Respecto de la vigencia de los Convenios Bilaterales, el art. 8 del reglamento de 2004 reitera que «en su campo de aplicación... sustituirá a cualquier otro convenio de Seguridad Social». Pese a ello la conocida y antes analizada doctrina jurisprudencial determina que parece aquélla sea la que se siga.

3. En cuanto a la exportación de las pensiones, se suprime la regla desterritorializadora posibilitando su adquisición o/y conservación con independencia del lugar en que se resida.

4. De la totalización de los periodos de carencia el reglamento de 2004 reproduce lo indicado en el previo 1243/72. La totalización comporta un sistema a cuyo través se conservan los derechos en vías de adquisición por medio de la adición de periodos de seguro (alta) y la principal 
vía no es otra que la de acumular todos los periodos computados por las distintas legislaciones nacionales.

5. Liquidación de las pensiones. Tanto el anterior como el actual Reglamento elaboran el sistema de liquidación haciendo jugar los principios de totalización y prorrateo.

El Reglamento de 2004 constituye una excelente plasmación de las tendencias armonizadoras y de mejora en el régimen de coordinación existente, lo que no significa que estén las cuestiones resueltas de forma pacífica y que no se mantengan las diferencias en los diferentes sistemas de Seguridad Social de los Países miembros. Situación que conlleva la necesidad de seguir trabajando en la línea de conseguir la armonización, y de simplificar las normas como se mantendrá a continuación.

\section{Perspectivas de futuro}

Los sistemas de protección social de los Estados miembros tienen ante sí diversos retos comunes y de indudable importancia, por ejemplo, la necesidad de adaptarse a la evolución del mundo del trabajo, las nuevas estructuras familiares y los espectaculares cambios demográficos que se prevén para los próximos decenios. Y al mismo tiempo, deben hallar el equilibrio entre los ciudadanos comunitarios de mantener un alto nivel de protección social y la necesidad de que los servicios públicos sean eficientes y respeten la disciplina presupuestaria. En los tiempos presentes, los acontecimientos económicos y políticos hacen que esta modernización sea más perentoria que nunca. Nos referimos a la disciplina macroeconómica que la estabilidad y crecimiento en la Unión Económica y Monetaria exigen; al compromiso de que los regímenes de protección social resulten más propicios para la creación del empleo, según se prevé en la estrategia Europea de Empleo y, a la necesidad de confirmar qué lugar debe ocupar la protección social entre los valores comunes de la Unión Europea.

Por lo antes expuesto el Consejo y el Consejo Europeo han venido realizando sucesivos mandatos respecto de la protección social. Los mandatos aludidos son los siguientes:

La resolución del Consejo de diciembre de 1999 sobre la modernización y la mejora de la protección social donde se destacó la necesidad de cooperación en la labor de modernización de la protección social, una cooperación basada en un diálogo estructurado y continuo, en el seguimiento e intercambio de información, experiencia y buenas prácticas entre los Estados miembros (punto 3 de las Conclusiones de 17 de diciembre); 
la necesidad de establecer un mecanismo de cooperación basado en los trabajos de un grupo de funcionarios de alto nivel y la necesidad de crearlo de forma inmediata (punto 11 y 12 de las Conclusiones).

La Decisión del Consejo de junio de 2000, en la que se establecía como uno de los cuatro objetivos generales y uno de los dos objetivos prioritarios para la modernización: garantizar la viabilidad y sostenibilidad de los regímenes de pensiones. Asimismo hacía hincapié en que «debería prestarse especial atención a la sostenibilidad de los regímenes de pensiones mediante la definición de dos líneas de acción. Con objeto de pronosticar mejor las tendencias futuras y lograr un conocimiento profundo de las estrategias nacionales recientes, aplicadas o previstas, de reforma de las pensiones». Se crea con esta misión un Comité de Protección social destinado a sustituir al Grupo de alto nivel.

La cooperación política en materia de protección social experimentó un avance significativo cuando el Consejo Europeo de Lisboa estableció una estrategia para la década que finalizará en 2010. Las conclusiones de Lisboa concedían una considerable importancia a la contribución de los sistemas de protección social a la consecución de nuevos objetivos estratégicos.

El enfoque de la protección social como elemento importante de las políticas económicas generales se reforzó con el Consejo Europeo de Niza y su Agenda de política social cuya idea básica la constituía el triángulo de políticas involucradas en la creación de un círculo virtuoso de políticas económicas, de empleo y de protección social, que confirmó el objetivo de modernizar y mejorar la protección social para que pudiera «responder a la transformación hacia la economía del conocimiento y al cambio de las estructuras sociales y familiares y apostar por el papel de la protección social como factor productivo».

Los mandatos y orientaciones del Consejo de Lisboa y de Niza han proporcionado el marco de los trabajos ya realizados y, de los pendientes según el calendario establecido a realizar según los objetivos para la modernización definidos en las Conclusiones del Consejo de diciembre de 1999 sobre la base de una Comunicación de la Comisión y que eran: hacer que trabajar sea rentable y garantizar unos ingresos seguros; conseguir pensiones seguras y sistemas de pensiones sostenibles; promover la inclusión social; y garantizar una atención sanitaria viable y de alta calidad. Objetivos que junto con la directrices de las políticas de empleo y económicas deberán permanecer estables hasta 2009. La validez de este marco se confirmó con los sucesivos mandatos de los Consejos Europeos siguientes que han fomentado el desarrollo de un nuevo objetivo o ambición en el marco europeo en los ámbitos de la inclusión social y las pensiones con el fin de fijar los indicadores comunes que reflejen los objetivos 
comunes, resultado que deberá presentarse en 2006 y, posteriormente los Estados miembros realizarán informes primero sobre la estrategia a seguir (en 2006) y, posteriormente sobre la aplicación de las estrategias (en los ejercicios 2007 y 2008). Será necesario esperar a ese momento para saber cuál va a ser el destino común de la protección social en la Unión Europea.

Pese a ello el futuro de la protección social en el marco europeo vendrá por intentar profundizar en la coordinación entre los Estados miembros al contribuir a garantizar la cohesión entre los Estados miembros y a la obtención de mejores resultados económicos. Para ello se hace necesario contar con una hacienda pública saneada y en la reforma del mercado laboral. De ahí que las tendencias, la estrategia europea necesariamente deberá centrarse en las medidas que redunden en beneficio del empleo. Así aumentando las personas acogidas a las medidas activas que mejoren las posibilidades de empleo y a la creación de empleo.

Las medidas de activación se deben centrar en la reforma de las prestaciones a fin de ofrecer incentivos a los perceptores para que busquen trabajo. En la reforma fiscal para favorecer la creación de empleo por los empresarios. En la adopción de las medidas tendentes a que los trabajadores de más edad continúen en activo y por ello invertir la tendencia europea de utilizar la jubilación anticipada que inducen a abandonar el mercado laboral. En elevar la edad de jubilación legalmente establecida y flexibilizando los tiempos de trabajo. En minorar los trámites necesarios para la creación de empleo autónomo. En el abaratamiento de los costes de personal. En la adopción de las medidas favorables al entorno familiar, apoyo familiar y conciliación (no tan sólo por aplicación del principio de igualdad sino además al ser una necesidad económica a la vista de la evolución demográfica y envejecimiento de la población) y, en la reforma de los sistemas de protección para reflejar la aparición de formas de actividad laboral (temporal, parcial).

Todas ellas teniendo naturalmente en cuenta el equilibrio financiero de los sistemas de Seguridad Social, como consecuencia del objetivo de tener una Hacienda saneada. El equilibrio se conseguirá y, por eso deberán constituir directrices europeas, la de aumentar los recursos y la de controlar el gasto (actual y futuro) de las pensiones.

La forma óptima de constituir más recursos para los regímenes de pensiones es la de aumentar el número de cotizantes y, por ello en directa relación al incremento de la tasa de empleo, de la población activa, e incluso desarrollar otras fuentes de financiación (recursos fiscales) para cubrir determinados elementos de las pensiones o prestaciones (ejemplo de la asistencia sanitaria y de los complementos por mínimos en el Sistema de Seguridad Social español). 
El control del gasto en materia de pensiones, debe contenerse aunque teniendo en cuenta las promesas en materia de pensiones y las expectativas de quienes proyectaron su retiro con arreglo a ellas. Las medidas que pueden generar importantes ahorros son la de aumentar la edad de jubilación, adecuar la revalorización de las pensiones al coste de la vida, calcular la pensión no en función de determinadas cotizaciones, sino en función de los ingresos percibidos en el pasado, etc.

La Unión Europea no pone en entredicho el principio, ya consagrado, según el cual cada Estado miembro tiene la responsabilidad de organizar y financiar su propio sistema. Sin embargo, junto a la concepción de políticas económicas coherentes que se sustenten mutuamente, se hace necesario un planteamiento concertado de modernización de la protección social. Y se hace necesario su coordinación y equiparación dado el volumen que el gasto en esta materia existe en la UE (aunque España sea el último de la cola).

Resulta evidente que la protección social es un componente fundamental del modelo social europeo al que se destina sobre un 27,5\% del PIB comunitario y que se destina en su mayor parte a pensiones. Además que la transformación del entorno económico que ha provocado la U.E. tendrá consecuencias para las estrategias de actuación en materia de crecimiento, aumento del empleo y estabilidad de precios. Por ello y por el grado de interdependencia entre las economías de los Estados miembros que se deriva de la U.E., la protección social está pasando a ser cada vez más un tema de interés común para los Estados miembros a los que interesa hallar cuál debe ser el planteamiento común, al encontrarse todos los Estados con problemas semejantes como son el envejecimiento demográfico y exigencia común de mayores niveles de cobertura.

La situación antes descrita lleva a que tengamos que plantearnos cuestiones tales como:

- ¿Ofrecen los regímenes de pensiones de vejez prestaciones demasiado generosas en caso de jubilación anticipada?

- ¿Está hoy justificada la aplicación de coeficientes reductores en determinados trabajos y categorías?

- ¿A los desempleados de mayor edad se les empuja a que busquen trabajo?

- ¿A las personas con problemas de salud se les ayuda a buscar otro trabajo? (aplicación de la doctrina administrativa relativa al artículo 143 de la LGSS en base a la que decide que la realización de actividad laboral posterior a la demanda habilita para su deducción por la TGSS).

- ¿Constituyen las prestaciones de muerte y supervivencia un desincentivo para buscar trabajo? (Las últimas reuniones de trabajo 
sobre esta materia incluso ha valorado suprimirla si el cónyuge supérstite estaba en activo).

- ¿Recompensa el sistema la prolongación de la vida activa más allá de la edad reglamentaria?

- ¿Existe suficiente protección a la familia? (no se ha desarrollado después de dos años las previsiones contenidas en la Ley de Familias Numerosas).

- ¿Y a la conciliación de la vida familiar? (posible creación de un permiso de paternindad tras el nacimiento).

- En definitiva y mientras se elaboran los estudios encomendados por el Comité de protección social, se precisa que se sigan realizando informes sobre aspectos de las políticas de pensiones a fin de intensificar y reforzar el intercambio y la comprensión entre los Estados miembros, esperando que la próxima reforma se lleve a cabo de manera integrada y coordinada.

Entre tanto, y en relación con los Reglamentos Comunitarios, las nuevas propuestas de la Comisión [vid. COM (2003) 468 final] se han visto limitadas a actualizar los mismos, e incluir los cambios que se han producido en las legislaciones nacionales, aclarar la situación jurídica y tener en cuenta los últimos cambios de la jurisprudencia del Tribunal de Justicia, así como a cuestiones relativamente marginales, tales como la determinación de la legislación de Seguridad Social aplicable en el caso de los asistentes parlamentarios europeos [vid. COM (2001) 344 final], o propuestas de carácter técnico [COM (2003) 378], tales como la dirigida a establecer las modalidades técnicas de introducción de la tarjeta sanitaria europea. 